\title{
Analysis and Comparative Study of Source Separation Performances in Feed-Forward and Feed-Back BSSs Based on Propagation Delays in Convolutive Mixture
}

\author{
Akihide HORITA Kenji NAKAYAMA Akihiro HIRANO \\ Graduate School of Natural Science and Technology, Kanazawa University \\ Kakuma-machi, Kanazawa, 920-1192 JAPAN \\ E-mail: horita@leo.ec.t.kanazawa-u.ac.jp, nakayama@t.kanazawa-u.ac.jp
}

\begin{abstract}
Feed-Forward (FF-) and Feed-Back (FB-) structures have been proposed for Blind Source Separation (BSS). The FF-BSS systems have some degrees of freedom in the solution space, and signal distortion is likely to occur in convolutive mixtures. On the other hand, the FBBSS structure does not cause signal distortion. However, it requires a condition on the propagation delays in the mixing process. In this paper, source separation performance in the FB-BSS is theoretically analyzed taking the propagation delays into account. Simulation is carried out by using white signals and speech signals as the signal sources. The FF-BSS system and the FB-BSS system are compared. Even though the FB-BSS can provide good separation performance, there exits some limitation on location of the signal sources and the sensors.
\end{abstract}

\section{Introduction}

Two kinds of approaches have been proposed for Blind Source Separation (BSS). One of them is a Feed-Forward (FF-) BSS, and the other is a Feed-Back (FB-) BSS [1]-[3]. In the FF-BSS, there exits some degrees of freedom in determining a separation block, and signal distortion is likely caused. Several methods have been proposed to suppress the signal distortion [4],[6],[7],[8]. Mainly, additional conditions are imposed on the learning process for source separation. On the other hand, the FB-BSS has no degree of freedom, and distortion free outputs can be obtained as a result of source separation. Therefore, the FB-BSS can provide good performances in both source separation and signal distortion. However, the FB-BSS requires some condition on propagation delays in a mixing process [5]. The source separation performance is degraded if the propagation delays do not satisfy this condition.

In this paper, source separation performance of the FB-BSS is theoretically analyzed based on the propagation delays. Simulation results obtained by using white signals and speech signals will be shown to confirm the theoretical analysis and to compare the FF-BSS and the FB-BSS. Finally, usefulness of the FB-BSS is discussed based on relative locations of the sources and the sensors. 


\section{Feed-Forward BSS}

\subsection{Network and Input-Output Equations}

A block diagram of the FF-BSS is shown in Fig.1(Left). In the separation block, an FIR filter, shown in Fig.1(Right), is used.

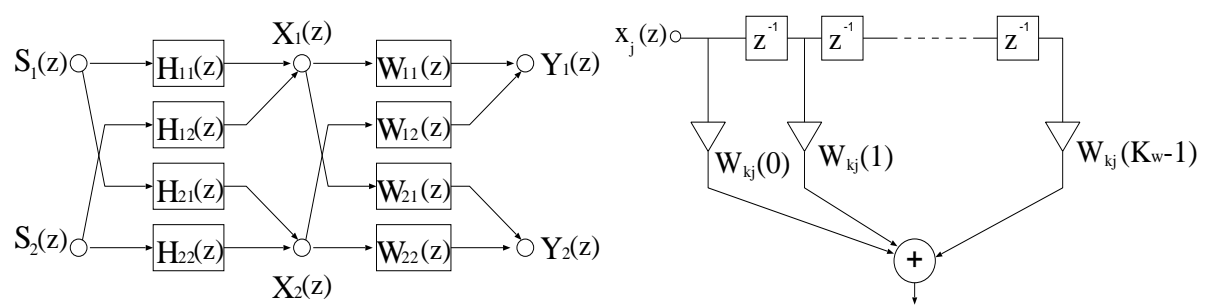

Fig. 1. (Left) A block diagram of FF-BSS. (Right) FIR filter used for $W_{k j}(z)$.

The sources $s_{i}(n), i=1,2, \cdots, N$ are convoluved with impulse responses of the mixing block $h_{j i}(n)$, and are observed as $x_{j}(n), j=1,2, \cdots, N$. The separation block outputs $y_{k}(n)$ are convolution sums of $w_{k j}(n)$ and $x_{j}(n)$.

$$
\begin{aligned}
& x_{j}(n)=\sum_{i=1}^{N} \sum_{m=0}^{K_{h}-1} h_{j i}(m) s_{i}(n-m) \\
& y_{k}(n)=\sum_{j=1}^{N} \sum_{l=0}^{K_{w}-1} w_{k j}(l) x_{j}(n-l)
\end{aligned}
$$

\subsection{Learning Algorithm}

The conventional learning algorithm [2],[3] is applied. $w_{k j}(n, l)$ are updated by

$$
\begin{aligned}
w_{k j}(n+1, l) & =w_{k j}(n, l)+\Delta w_{k j}(n, l) \\
\Delta w_{k j}(n, l) & =\eta\left\{w_{k j}(n, l)-\sum_{q=0}^{K_{w}-1} \varphi\left(y_{k}(n)\right) y_{p}(n-l+q) w_{p j}(n, q)\right\}, p \neq j(4)
\end{aligned}
$$

$\varphi\left(y_{k}(n)\right)$ is a probability density function of $y_{k}(n)$. In the above algorithm, the signal distortion is likely caused. A technique to suppress the signal distortion has been proposed [8]. First, $w_{k j}(n+1, l)$ are updated following Eqs.(3) and (4). Second, $w_{j j}(n+1, l)$ are modified as follows:

$$
w_{j j}(n+1, l)=(1-\alpha) \tilde{w}_{j j}(n+1, l)+\alpha \bar{w}_{j j}(n+1, l), 0<\alpha \leq 1
$$

$\tilde{w}_{j j}(n+1, l)$ are updated by Eqs. $(3)$ and $(4) \cdot \bar{w}_{j j}(n+1, l)$ are determined by the conditions of complete source separation and signal distortion free [8].

\section{Feed-Back BSS}

\subsection{Block Diagram and Input-Output Relations}

A block diagram of the FB-BSS is shown in Fig.2(Left) [1]. The separation block employs an FIR filter shown in Fig.2(Right). 


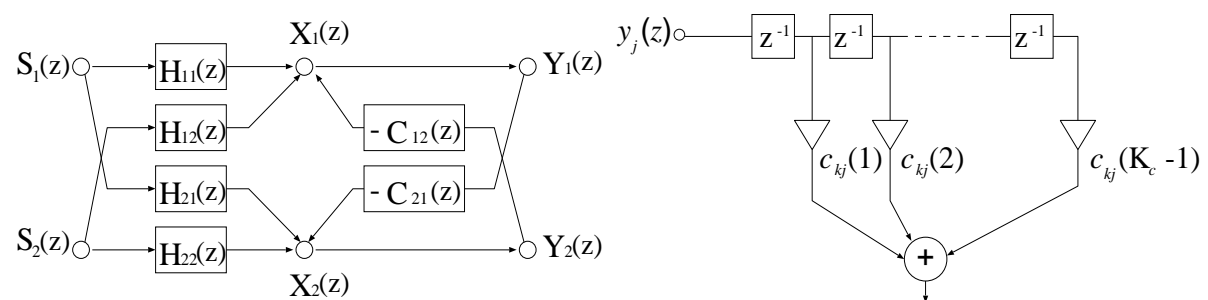

Fig. 2. (Left) Block diagram of FB-BSS. (Right) FIR filter used for $C_{21}(z)$ and $C_{12}(z)$

Since a feed-back loop in a discrete time system needs at least one sample delay, a direct path from the input to the output is not used in the FIR filter, that is $c_{k j}(0)=0$. The outputs of the separation block are expressed by

$$
y_{k}(n)=x_{k}(n)-\sum_{\substack{j=1 \\ \neq k}}^{N} \sum_{l=1}^{K_{c}-1} c_{k j}(l) y_{j}(n-l)
$$

\subsection{Learning Algorithm}

The update equation of $c_{k j}(n, l)$ was proposed by Jutten et al by using the Kullback-Leibler mutual information, and is shown here [1]. $f\left(y_{k}(n)\right)$ and $g\left(y_{j}(n-\right.$ $l)$ ) are different odd functions. One of them is at least a nonlinear function.

$$
\begin{aligned}
c_{k j}(n+1, l) & =c_{k j}(n, l)+\Delta c_{k j}(n, l) \\
\Delta c_{k j}(n, l) & =\mu f\left(y_{k}(n)\right) g\left(y_{j}(n-l)\right)
\end{aligned}
$$

\section{Derivation of Learning Algorithm for FB-BSS Based on Propagation Delay}

A learning algorithm is derived based on the propagation delays in the mixing process [5]. For simplicity, the FB-BSS having 2 sources, 2 sensors and 2 outputs, as shown in Fig.2(Left), is taken into account. It is assumed that the propagation delays of $H_{11}(z)$ and $H_{22}(z)$ are less than those of $H_{21}(z)$ and $H_{12}(z)$. This means that the sensor of $X_{1}(z)$ locates close to $S_{1}(z)$, and that of $X_{2}(z)$ locates close to $S_{2}(z)$. Thus, this assumption is actually acceptable.

The outputs of the separation block can be expressed by [5]

$$
\begin{aligned}
& {\left[\begin{array}{l}
Y_{1}(z) \\
Y_{2}(z)
\end{array}\right]=\frac{1}{1-C_{12}(z) C_{21}(z)}\left[\begin{array}{cc}
1 & -C_{12}(z) \\
-C_{21}(z) & 1
\end{array}\right]\left[\begin{array}{l}
H_{11}(z) H_{12}(z) \\
H_{21}(z) H_{22}(z)
\end{array}\right]\left[\begin{array}{l}
S_{1}(z) \\
S_{2}(z)
\end{array}\right] } \\
= & \frac{1}{1-C_{12}(z) C_{21}(z)}\left[\begin{array}{l}
H_{11}(z)-C_{12}(z) H_{21}(z) H_{12}(z)-C_{12}(z) H_{22}(z) \\
H_{21}(z)-C_{21}(z) H_{11}(z) H_{22}(z)-C_{21}(z) H_{12}(z)
\end{array}\right]\left[\begin{array}{l}
S_{1}(z) \\
S_{2}(z)
\end{array}\right]
\end{aligned}
$$


When source separation is complete, $C_{k j}(z)$ and $y_{k}(n)$ have the following two kinds of solutions (a) and (b).

$$
\begin{aligned}
& \boldsymbol{h}_{j i}=\left[h_{j i}(0), h_{j i}(1), \cdots, h_{j i}\left(K_{h}-1\right)\right]^{T} \\
& \boldsymbol{s}_{i}(n)=\left[s_{i}(n), s_{i}(n-1), \cdots, s_{i}\left(n-K_{h}+1\right)\right]^{T}
\end{aligned}
$$

(a) Non-diagonal elements are zero.

$$
\begin{array}{ll}
C_{12}(z)=\frac{H_{12}(z)}{H_{22}(z)} & C_{21}(z)=\frac{H_{21}(z)}{H_{11}(z)} \\
y_{1}(n)=\boldsymbol{h}_{11}^{T} \boldsymbol{s}_{1}(n) & y_{2}(n)=\boldsymbol{h}_{22}^{T} \boldsymbol{s}_{2}(n)
\end{array}
$$

(b) Diagonal elements are zero.

$$
\begin{array}{ll}
C_{12}(z)=\frac{H_{11}(z)}{H_{21}(z)} & C_{21}(z)=\frac{H_{22}(z)}{H_{12}(z)} \\
y_{1}(n)=\boldsymbol{h}_{12}^{T} \boldsymbol{s}_{2}(n) & y_{2}(n)=\boldsymbol{h}_{21}^{T} \boldsymbol{s}_{1}(n)
\end{array}
$$

From the assumption regarding the delay of $H_{j i}(z), C_{21}(z)$ and $C_{12}(z)$ in $(a)$ have a positive delay, that is, they are causal systems. On the other hand, $C_{21}(z)$ and $C_{12}(z)$ in $(b)$ have a negative delay, resulting in non-causal systems, which cannot be realizable. For this reason, $S_{1}(z)$ cannot be cancelled at $X_{1}(z)$. In the same manner, $S_{2}(z)$ cannot be cancelled at $X_{2}(z)$. This means that the diagonal elements of Eq.(9) cannot be cancelled by adjusting $C_{j k}(z)$. On the other hand, $S_{2}(z)$ and $S_{1}(z)$ can be cancelled at $X_{1}(z)$ and $X_{2}(z)$, respectively. In other words, the non-diagonal elements of Eq.(9) can be cancelled. Combining these properties, the power of the separation block outputs can be set as a cost function. Applying the gradient method, the same learning algorithm as shown in Eqs.(7) and (8) can be derived [5].

\section{Analysis of Source Separation Based on Propagation Delay in Mixing Process}

\subsection{Effects of Propagation Delay on Learning FB-BSS}

It is also assumed that, in the FB-BSS shown in Fig.2, $S_{1}(z)$ and $S_{2}(z)$ are separated at $Y_{1}(z)$ and $Y_{2}(z)$, respectively. This does not lose generality. Source separation performance of the FB-BSS is determined by the following conditions.

1. $S_{2}(z)$ and $S_{1}(z)$ should be cancelled at $X_{1}(z)$ and $X_{2}(z)$, respectively.

2. $S_{1}(z)$ and $S_{2}(z)$ should be preserved at $X_{1}(z)$ and $X_{2}(z)$, respectively.

As discussed in Sec.4, the learning of the FB-BSS is equivalent to minimize the output powers. If the delay of $C_{12}(z) H_{21}(z)$ is large enough compared to that of $H_{11}(z)$, then the $S_{1}(z)$ component cannot be cancelled at $X_{1}(z)$, and can be separated at $Y_{1}(z)$. This means that the power of $Y_{1}(z)$ can be minimized by cancelling the $S_{2}(z)$ components at $X_{1}(z)$. Situation is the same as in minimizing the power of $Y_{2}(z)$. In these cases, $C_{k j}(z)$ converge to the optimal solutions given by Eq.(12). When difference between the delays of $C_{12}(z) H_{21}(z)$ and $H_{11}(z)$ is not large enough, correlation between $C_{12}(z) H_{21}(z) S_{1}(z)$ and $H_{11}(z) S_{1}(z)$ will 
be increased. As a result, some cancellation between them can be possible. In other words, the power of $Y_{1}(z)$ can be minimized by reducing not only the $S_{2}(z)$ component but also the $S_{1}(z)$ component. In this situation, $C_{k j}(z)$ are shifted from the optimal solutions given by Eq.(12) toward the undesirable solutions given by Eq.(14), resulting in poor source separation performances.

First, the condition (1) is taken into account. As shown in Fig.2, the transfer functions $C_{k j}(z)$ require at least one sample delay, corresponding to $z^{-1}$. Therefore, in order to cancel $S_{2}(z)$ at $X_{1}(z)$, the difference between the delays of $H_{21}$ and $H_{11}(z)$ should be equal to or larger than one sample delay. If this condition is not sufficiently satisfied, $S_{2}(z)$ cannot be well cancelled. The same situation is held in $X_{2}(z)$.

Next, the condition (2) is taken into account. Preserving the $S_{1}(z)$ component in $X_{1}(z)$, at the same time, in $Y_{1}(z)$, is highly dependent on the difference between the delays of $C_{12}(z) H_{12}(z)$ and $H_{11}(z)$. Here, the signal is simply denoted $u(n)$ for convenience. The sampling frequency of $u(n)$ is denoted $f_{s}$. In order to consider the delay difference less than the sampling period $T=1 / f_{s}$, the sampling frequency $f_{s}$ is converted to $K f_{s}, K>1$. This up-sampling is carried out by inserting zero samples and band limitation. Let $u_{z}(n)$ be the up-sampled signal by inserting $K-1$ zero samples between the $u(n)$ samples. The frequency component of $u(n)$ is assumed to be distributed in $0 \sim f_{s} / m . u_{z}(n)$ is band limited by using the ideal filter, whose pass band is $0 \sim f_{s} / m$ and the sampling frequency is $K f_{s}$. An impulse response of this ideal filter is given by

$$
\phi(n)=\frac{2}{K m} \frac{\sin \left(\frac{2 \pi}{K m} n\right)}{\frac{2 \pi}{K m} n}
$$

$\phi(n)$ is regarded as an interpolation function. Let the band limited signal be $u_{K}(n)$, which is a convolution sum of $u_{z}(n)$ and $\phi(n)$ as shown below.

$$
u_{K}(n)=\sum_{k=0}^{n} \phi(n-k) u_{z}(k)
$$

\subsection{Canceling $u_{K}(n)$ by Using $u_{K}(n-l)$}

In this case, the delay difference is $l$ samples under the sampling frequency of $K f_{s}$. We will consider cancelation of $S_{1}(z)$ at $X_{1}(z)$ depending on delay difference. In this case, $u_{K}(n)$ and $u_{K}(n-l)$ can be regarded as $h_{11}(n) * s_{1}(n)$ and $c_{12}(n) * h_{21}(n) * s_{1}(n)$, respectively. The operation $*$ is a convolution sum. Since amplitude and phase responses of the signals can be adjusted by $C_{j k}(z)$, an effect of the delay difference is only taken into account in this section.

Cancelation of $u_{K}(n)$ by using $u_{K}(n-l)$ can be expressed by

$$
\begin{aligned}
u_{K}(n)-u_{K}(n-l) & =\sum_{k=0}^{n} \phi(n-k) u_{z}(k)-\sum_{k=0}^{n} \phi(n-k-l) u_{z}(k) \\
& =\sum_{k=0}^{n}[\phi(n-k)-\phi(n-k-l)] u_{z}(k)
\end{aligned}
$$


From the above equation, the cancelation of $u_{K}(n)$ can be evaluated by $[\phi(n-k)-\phi(n-k-l)]$, which is equivalent to $\phi(l) . \phi(l)$ becomes zero at $l=K m / 2$, and takes small value after that. Therefore, as a rule of thumb, the delay difference, with which $u_{K}(n)$ can be cancelled by using $u_{K}(n-l)$, is less than a $K m / 2$ sample delay under the sampling frequency of $K f_{s}$, which is equivalent to an $m / 2$ sample delay with the $f_{s}$ sampling frequency.

An example of $\phi(n)$ is shown in Fig. 3 , in which $m=4$ and $K=4$. In this

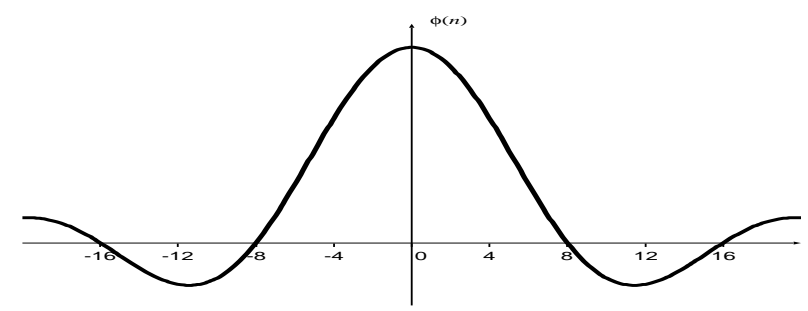

Fig. 3. Example of interpolation function $\phi(n)$ with $m=4$ and $K=4$.

figure, one scale on the horizontal axis indicates $T / K$ sec and $4(=K)$ scales corresponds to $T$ sec. $\phi(n)$ takes zero at 8 samples $(\times T / K)$ and 2 samples $(\times T)$, and takes small values after these points.

(Example 1)

Let $f_{s}=8 \mathrm{kHz}$ and the frequency components of the signal are distributed under $1 \mathrm{kHz}$. Since $f_{s} / m=1 k H z$ and $m=8$, if the delay difference between $C_{12}(z) H_{12}(z)$ and $H_{11}(z)$ is larger than $m / 2=4$ samples, then cancellation of $S_{1}(z)$ at $X_{1}(z)$ is small. Since $C_{k j}(z)$ have 1 sample delay, by assigning 3 samples delay between $H_{21}(z)$ and $H_{11}(z)$, the above conditions (1) and (2) can be simultaneously satisfied. In this case, the source separation performance is mainly determined by the condition(2).

(Example 2)

Let $f_{s}=8 \mathrm{kHz}$ and the frequency component of the signal is distributed under $4 \mathrm{kHz}$. Since $f_{s} / m=4 \mathrm{kHz}$ and $m=2$, if the delay difference between $C_{12}(z) H_{12}(z)$ and $H_{11}(z)$ is more than $m / 2=1$ samples, then the cancelation of $S_{1}(z)$ at $X_{1}(z)$ is small. Since $C_{k j}(z)$ has 1 sample delay, the delay difference between $H_{21}(z)$ and $H_{11}(z)$ is not necessary. However, in order to satisfy the condition(1), at least 1 sample delay is required. In this case, the source separation performance is mainly determined by the condition(1).

\subsection{Cancelation of $\boldsymbol{u}_{\boldsymbol{K}}(\boldsymbol{n})$ Evaluated by Correlation}

The cancelation of $u_{K}(n)$ by using $u_{K}(n-l)$ can be also evaluated by the mean square of $\phi(n-k)-\phi(n-k-l)$ as follows:

$$
\begin{aligned}
& E\left[(\phi(n-k)-\phi(n-k-l))^{2}\right] \\
& =E\left[\phi^{2}(n-k)\right]-2 E[\phi(n-k) \phi(n-k-l)]+E\left[\phi^{2}(n-k-l)\right]
\end{aligned}
$$

The delay difference of interest is a few samples delay, that is $l$ is a small integer. Therefore, $E\left[\phi^{2}(n-k)\right]$ and $E\left[\phi^{2}(n-k-l)\right]$ are almost the same. They are 
denoted $E\left[\phi^{2}(n-k)\right]$ here. The above equation is further rewritten as

$$
\begin{aligned}
& 2 E\left[\phi^{2}(n-k)\right]-2 E[\phi(n-k) \phi(n-k-l)] \\
& =2 E\left[\phi^{2}(n-k)\right]\left(1-\frac{E[\phi(n-k) \phi(n-k-l)]}{E\left[\phi^{2}(n-k)\right]}\right)
\end{aligned}
$$

Since $E\left[\phi^{2}(n-k)\right]$ is the signal power, it is not related to the cancelation of $u_{K}(n)$. From the above equation, if a correlation of $u_{K}(n-k)$ and $u_{K}(n-k-l)$, that is $E[\phi(n-k) \phi(n-k-l)] / E\left[\phi^{2}(n-k)\right]$ is close to 1 , then $S_{1}(z)$ is well cancelled at $X_{1}(z)$. Simulations in Sec.6.2, the correlation of $h_{11}(n) * s_{1}(n)$ and $h_{21}(n) * s_{1}(n)$ is used to evaluate the cancelation of $S_{1}(z)$ at $X_{1}(z)$.

On the contrary, in the FF-BSS, the propagation delays in the mixing process do not affect the source separation performance.

\subsection{Effects of Fractional Propagation Delays}

The propagation delay in the mixing process is not always an integer times of the sampling period $T=1 / f_{s}$. It may be sometime a fractional period. This means the transfer functions $H_{j i}(z)$ in the mixing process cannot be expressed by a rational function of $z^{-1}$. On the other hand, the FIR filters used in the separation block are implemented with the sampling frequency of $f_{s}=1 / T$, and their transfer function are expressed by a power series of $z^{-1}$. Therefore, if the propagation delay is not an integer times of $T$, the FIR filter cannot realize the inverse function of the mixing process, as a result, the source separation performance is degraded. This point is also investigated through simulation.

\section{Simulations and Discussions}

\subsection{Simulation Setup}

2-channel and 3-channel models are used. Simulation results only for the 2channel model are demonstrated due to page limitation. The sampling frequency is $f_{s}=8 \mathrm{kHz}$. White signals and speech signals, whose spectrum is shown in Fig.4(Left), are used as the sources. Figure 4(Right) shows a mixing process.
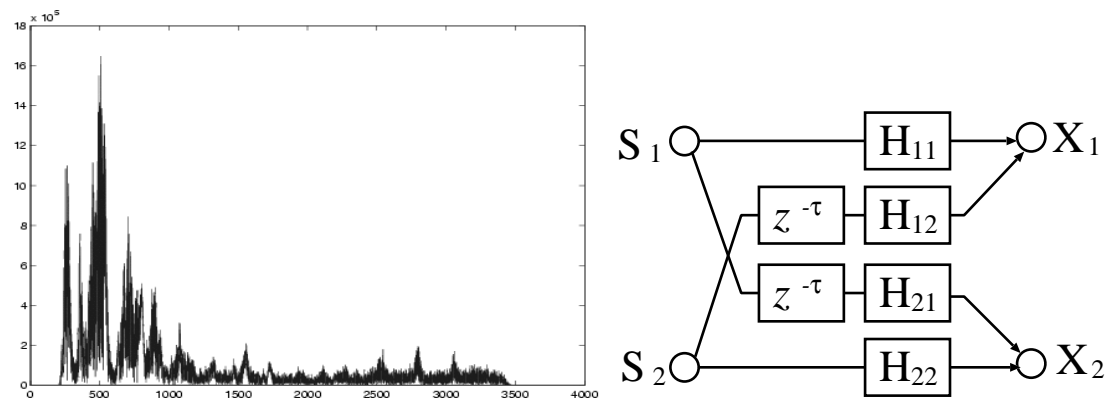

Fig. 4. (Left) Spectrum of speech signal. (Right) Mixing process with delay difference.

The delay difference between $H_{j j}(z)$ and $H_{j i}(z), j \neq i$ is realized by $\tau$. Two kinds models for $H_{j i}(z)$ are used. One of them is an instantaneous mixing process 
shown in Eq.(21) and the other is an approximately actual room environment. The source separation is evaluated by the Signal-to-Interference Ratio (SIR [dB]) given by Eq.(24). $A_{k i}(z)$ is a transfer function from the $i$ th signal source to the $k$ th output of the separation block.

$$
\begin{aligned}
\boldsymbol{H}(z) & =\left[\begin{array}{cc}
1 & 0.9 \\
0.9 & 1
\end{array}\right] \\
\sigma_{s}^{2} & =\frac{1}{2 \pi} \sum_{i=1}^{N} \int_{-\pi}^{\pi}\left|A_{i i}\left(e^{j \omega}\right) S_{i}\left(e^{j \omega}\right)\right|^{2} d \omega \\
\sigma_{i}^{2} & =\frac{1}{2 \pi} \sum_{k=1}^{N} \sum_{\substack{i=1 \\
\neq k}}^{N} \int_{-\pi}^{\pi}\left|A_{k i}\left(e^{j \omega}\right) S_{i}\left(e^{j \omega}\right)\right|^{2} d \omega \\
S I R & =10 \log _{10} \frac{\sigma_{s}^{2}}{\sigma_{i}^{2}}[d B]
\end{aligned}
$$

\subsection{Instantaneous and Propagation Delay Model}

Figure 5 shows the SIR and the correlation by using Eq.(21) and the white signal sources. 'Delay difference' means $\tau$ in Fig.4. $C_{12}(z)$ has one sample delay
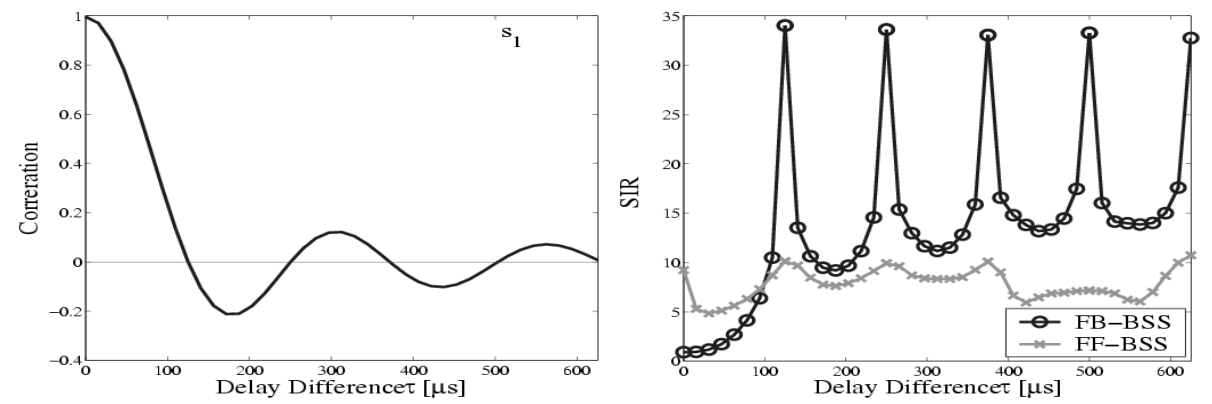

Fig. 5. White signal sources. (Left) Correlation between $h_{11}(n) * s_{1}(n)$ and $h_{21}(n) *$ $s_{1}(n)$. (Right) SIR with respect to $\tau$.

as shown in Fig.2(Right), the delay difference at $X_{1}(z)$ becomes $\tau+125 \mu s$. The cancelation of $S_{1}(z)$ at $X_{1}(z)$ should be evaluated by $\tau+125 \mu s$. The band width of the white signal is $4 \mathrm{kHz}$, and $m=2$ as discussed in Sec.5.1. The interpolation function is given by

$$
\phi(n)=\frac{1}{K} \frac{\sin \left(\frac{2 \pi}{2 K} n\right)}{\frac{2 \pi}{2 K} n}
$$

$\phi(n)$ takes zero at every $K$ samples, which correspond to one sample with the $8 \mathrm{kHz}$ sampling frequency. Since the white signals are sampled by $8 \mathrm{kHz}$, there is no correlation between the samples. The correlation shown in Fig.5(Left) is also similar to $\phi(n)$ given by Eq.(25). As described in Sec.5.1, the rule of thumb for the delay difference, with which the condition(2) is satisfied, is the $m / 2$ sample delay, which is one sample delay under the $8 \mathrm{kHz}$ sampling frequency. Since $C_{12}(z)$ 
and $C_{21}(z)$ include one sample delay, the condition(2) is satisfied by using $\tau=0$. However, the condition(1) requires $\tau \geq$ one sample delay. Therefore, the SIR converges to high level at $\tau=125 \mu \mathrm{s}$. Like this, in the case of the white signals, the condition(1) is dominant to achieve a high SIR. This situation is exactly the same as Example 2 in Sec.5.1.

The SIR has peaks at every $\tau=125 \mu \mathrm{s}$. This is due to no correlation at these points. Furthermore, the mixing process can be expressed as a rational function of $z^{-1}$, whose inverse function can be approximated by the separation block, which is implemented with the $8 \mathrm{kHz}$ sampling frequency.

\subsection{Convolutive and Propagation Delay Model}

The mixing process similar to actual audio environment is used. $H_{j i}(z)$ have no delay difference. The delay difference is also realized by $\tau$ in Fig.4.

Figure 6 shows simulation results for the speech signal sources. The correlation becomes zero around $400 \mu \mathrm{s}$. From the analysis in Sec.5.1, $125 \mu \mathrm{s} \times(\mathrm{m} / 2)=$ $400 \mu \mathrm{s}$, and $m \simeq 6.4$. This is due to the frequency component of the speech signals, which is mainly distributed under $8 \mathrm{kHz} / 6.4 \simeq 1.25 \mathrm{kHz}$ as shown in Fig.4(Left). The SIR is gradually increased until $\tau=350 \mu s$. The delay difference at $X_{1}(z)$ becomes $(350+125) \mu s=475 \mu s$, which approximately corresponds to $m / 2$ sample delay difference. In this case, the condition(2) is dominant. This case corresponds to Example 1 in Sec.5.1.
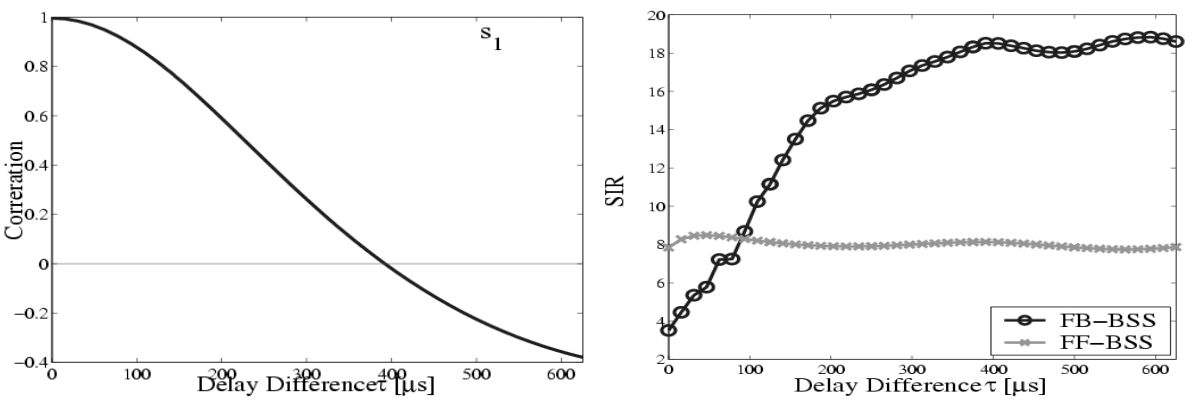

Fig. 6. Speech signal sources. (Left) Correlation between $h_{11}(n) * s_{1}(n)$ and $h_{21}(n) *$ $s_{1}(n)$. (Right) SIR with respect to $\tau$.

The SIR of the FF-BSS is not affected by the delay difference $\tau$, and it is almost constant with respect to $\tau$.

The simulation results for the 3-channel model are almost similar to those of the 2-channel model.

\subsection{Delay Difference Based on Location of Signal Sources and Sensors.}

A relation between the delay difference and location of the sources and the sensors is investigated taking sound propagation time into account. A layout of them shown in Fig.7(Left) is taken into account. Relations of the delay differences and the layouts are shown in Fig.7(Right). The FB-BSS can provide better performances with $\tau=125 \mu \mathrm{s}$, which is generated by $L_{2}=5,9,25 \mathrm{~cm}$ for $\left(L_{1}, D\right)=$ $(300,100) \mathrm{cm},(100,100) \mathrm{cm}$ and $(100,300) \mathrm{cm}$, respectively. 


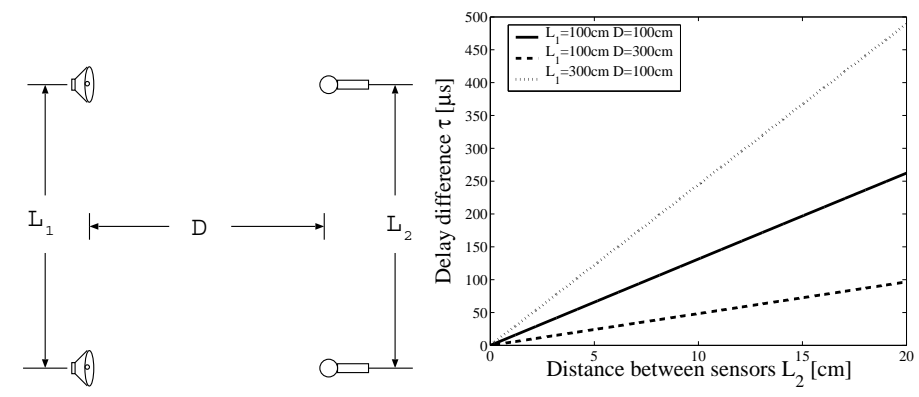

Fig. 7. (Left) Layout of signal sources and sensors. (Right) Delay difference $\tau$ depending on layout of signal sources and sensors.

\section{Conclusions}

The source separation performance of the FB-BSS is theoretically analyzed based on the propagation delay difference in the mixing process. It is determined by two conditions, canceling the interferences and preserving the desired source, which are highly dependent on the delay difference in the mixing process. Simulation results using white signal sources and speech signal sources support the theoretical analysis. Furthermore, a relation between the delay difference and the layout of the sources and sensors is discussed. The layout conditions, which are useful for the FB-BSS or the FF-BSS, are derived.

\section{References}

1. H.L.Nguyen Thi and C.Jutten, "Blind source separation for convolutive mixtures", Signal Processing, vol.45, no.2, pp.209229, March 1995.

2. A.Cichocki, S.Amari, M.Adachi, W.Kasprzak, "Self-adaptive neural networks for blind separation of sources", Proc. ISCAS'96, Atlanta, pp.157-161, 1996.

3. S.Amari, T.Chen and A.Cichocki, "Stability analysis of learning algorithms for blind source separation", Neural Networks, vol.10, no.8, pp.1345-1351, 1997.

4. N.Murata, S.Ikeda and A. Ziehe, "An approach to blind source separation based on temporal structure of speech signals", Neurocomputing, vol.41, pp.1-24, Oct. 2001.

5. K.Nakayama, A.Hirano and A.Horita, "A learning algorithm for convolutive blind source separation with transmission delay constraint", Proc. IJCNN'2002, pp.12871292, May 2002.

6. H.Saruwatari, T.Takatani, H.Yamajo, T.Sishikawa and K.Shikano, "Blind separation and deconvolution for real convolutive mixture of temporally correlated acoustic signals using SIMO-model-based ICA", ICA'03 pp.549-554 April 2003

7. A.Horita, K.Nakayama, A.Hirano and Y.Dejima, "Analysis of signal separation and signal distortion in feedforward and feedback blind source separation based on source spectra", IEEE\&INNS, Proc., IJCNN2005, Montreal, pp.1257-1262, JulyAug., 2005.

8. A.Horita, K.Nakayama, A.Hirano and Y.Dejima, "A learning algorithm with distortion free constraint and comparative study for feedforward and feedback BSS", Proc., EUSIPCO2006, Florence, Italy, Sept 2006. 